\title{
Preface to the Special Issue: Anode Materials for Rechargeable Batteries
}

\author{
Jianmin $\mathrm{Ma}^{1} \cdot \mathrm{Yu}-\mathrm{TaO} \mathrm{Li}^{2}$ \\ Accepted: 28 January 2021 / Published online: 22 February 2021 \\ (c) The Chinese Society for Metals (CSM) and Springer-Verlag GmbH Germany, part of Springer Nature 2021
}

Rechargeable Li-ion batteries were first commercialized in 1991 by Sony company, and the current portable electronic devices and electric vehicles powered by rechargeable batteries have significantly accelerated the development of social civilization. The Nobel Prize in Chemistry 2019 was also awarded jointly to John B. Goodenough, M. Stanley Whittingham, and Akira Yoshino for their outstanding contribution to rechargeable Li-ion batteries.

The rechargeable Li-ion batteries consist of anodes, cathodes, and electrolytes. During charge/discharge, the Li-ions transfer between the anode and cathode through the electrolytes, and the electrons move through the external circuit. The commonly used anode material, graphite, in commercial rechargeable Li-ion batteries can form a stable solid electrolyte interphase layer with liquid electrolytes, which increases the cycling life of the batteries. However, graphite anode can only accommodate one Li-ion with six carbon atoms and show a low capacity of $370 \mathrm{mAh} \mathrm{g}^{-1}$. Moreover, the lithium can be plated on the surface of graphite when the batteries are charged at a high current density because of the relatively low Li-ion diffusion coefficient; the uneven plated lithium can form lithium dendrites, penetrate the separator and short-circuit the batteries, thus resulting in serious safety problems. Therefore, it is still urgent to develop new anode materials for rechargeable batteries.

The anode materials usually store Li-ions through three different mechanisms, including intercalation/deintercalation, alloy, and conversion process. The intercalation/deinteracation anode materials such as spinel $\mathrm{Li}_{4} \mathrm{Ti}_{5} \mathrm{O}_{12}$ has a Li-ion transport channel, and their capacity at high current

Jianmin Ma

nanoelechem@hnu.edu.cn

1 School of Physics and Electronics, Hunan University, Changsha 410082, China

2 Materials Science and Engineering Program and Texas Materials Institute, The University of Texas At Austin, Austin, TX 78712, USA density is closely related to the morphology and surface area of the powders. The intercalation oxide anode materials also have a high charge/discharge voltage than the intercalation sulfides. Compared with the intercalation/deintercalation anodes, the anode such as $\mathrm{Si}, \mathrm{Al}$, and $\mathrm{SnO}_{2}$ can react with lithium metal to form alloys and show much higher capacity than the graphite and other kinds of anodes. However, the huge volume change of these alloy anodes during cycling destroys the solid electrolyte interphase layer, reduces the Coulombic efficiency and cycling life of the batteries. For the conversion anodes, they usually are low-cost oxide (e.g., $\mathrm{Fe}_{2} \mathrm{O}_{3}, \mathrm{MoO}_{2}, \mathrm{MoSe}_{2}$ ) and are environmentally friendly. However, the batteries with these conversion anodes usually have limited cycle performance because of the slow diffusion of Li-ions. Moreover, the large voltage hysteresis of the batteries significantly reduces the energy efficiency of the batteries.

Based on the challenges of developing new anode materials for rechargeable batteries, we have this special issue on anodes in rechargeable batteries. 16 papers from the active research groups in this area have been accepted for final publication in the issue. Most papers are about the anode in lithium batteries, and several papers on the Na-ion are also accepted because of the low cost of rechargeable sodium batteries. We sincerely thank all the authors for their valuable contribution to the area, and we also thank all the reviewers for their constructive comments, which greatly increase the papers' quality. We also appreciate the assistance from the editors and the publishing team of Acta Metallurgica Sinica (English letters), and we hope the issue could provide an insightful view of the development of new anode materails for rechargeable batteries. 


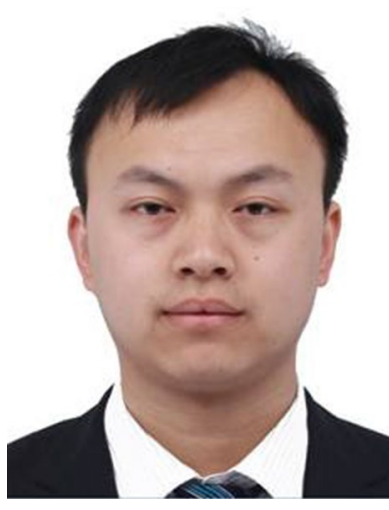

Jianmin Ma is the professor in the Hunan University, Changsha, China. He received his B.S. degree in Chemistry from the Shanxi Normal University in 2003 and Ph.D. degree in Materials Physics and Chemistry from Nankai University in 2011. During 2011-2015, he also conducted the research in several overseas universities as a postdoctoral research associate. $\mathrm{He}$ serves as the Academic Editor for Rare Metals, and editorial board members for Journal of Energy Chemistry, Chinese Chemical Letters, Nano-Micro Letters, Journal of Physics: Condensed Matter, and others. His research interest focuses on the energy storage devices and components including metal anodes and electrolytes, and theoretical calculations from Density Functional Theory and Molecular Dynamics to Finite Element Analysis.

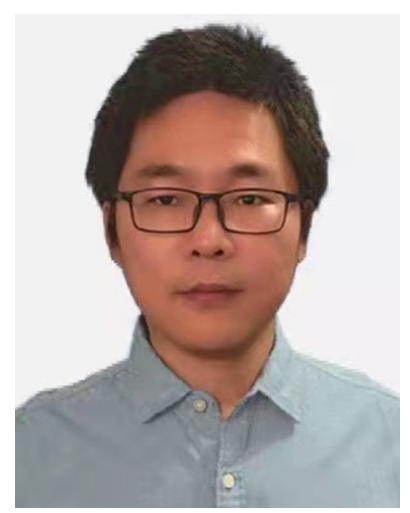

Yu-Tao Li is a research fellow at the University of Texas at Austin in the USA. He received his $\mathrm{Ph} . \mathrm{D}$. degree in Materials Science and Engineering from Tsinghua University in 2013. Now, he is working with Prof. John B. Goodenough to do the research work about transitional metal oxides and their application in batteries and catalysts. 